\title{
PHILelergict
}

(c) (1) \&) $९$ Philologica Canariensia 24 (2018), pp. 55-75

DOI: 10.20420/PhilCan.2018.197

Recibido: 12 de febrero de 2018; aceptado: 13 de junio de 2018

Publicado: 18 de julio de 2018

\section{ESTUDIO SOCIOLINGÜÍSTICO DEL DISCURSO REFERIDO COMO CATEGORÍA Y CONTINUUM EN EL ESPAÑOL HABLADO DE CARACAS*}

\author{
MARÍA JOSÉ GALLUCCI \\ Universidad Central de Venezuela / Universidad de Zaragoza
}

\begin{abstract}
RESUMEN: En este estudio consideramos el discurso referido (DR) como una categoría en la que se agrupan como un continuum diferentes formas. A partir del análisis de un conjunto de muestras orales del Corpus sociolingüístico de Caracas 2004-2013 -o PRESEEA-CA- (Bentivoglio y Malaver, 2006, 2012; Gallucci, González y Malaver, 2013) estratificadas según la edad, el sexo y el grado de instrucción de los hablantes, identificamos seis tipos principales de citas -que denominamos propias o propiamente de $D R-\mathrm{y}$ dos periféricas -o impropias-. Además de la clasificación y de los criterios metodológicos empleados para el análisis del DR en conversaciones semidirigidas, describimos su distribución en la muestra, con especial referencia a la cita directa, y en relación con las variables sociales antes descritas.
\end{abstract}

PALABRAS CLAVE: discurso referido, continuum, sociolingüística, PRESEEA, español de Caracas

Sociolinguistic study of reported speech as both a category and a continuum in the spoken Spanish of Caracas

\begin{abstract}
In this study we consider reported speech (RS) as a continuum of different forms. Based on the analysis of spoken discourse from Corpus sociolingüístico de Caracas 2004-2013 -or PRESEEA-CA- (Bentivoglio y Malaver 2006, 2012; Gallucci, González y Malaver, 2013), which is stratified by age, sex, and educational level, we identify six main types of quotes -which we call proper of $R S$ - and two peripheral types -or improper-. In addition to our classification and methodological criteria for the analysis of RS in semi-structured conversations, we describe its distribution on the sample, with special reference to the direct speech and to the social variables described above.
\end{abstract}

KEYWORDS: reported speech, continuum, sociolinguistics, PRESEEA, Spanish spoken in Caracas

\section{INTRODUCCIÓN}

La reflexividad lingüística es la propiedad del lenguaje que hace posible que los hablantes puedan reproducir -o referir- las palabras propias o ajenas que efectivamente tuvieron lugar o que son imaginarias, como cuando el locutor las pone en boca de otro aunque no hayan sido efectivamente pronunciadas. ${ }^{1}$ Mediante esta propiedad, que se materializa a través de diversos 
canales semióticos, tales como marcas prosódicas o animaciones (cf. Niemelä, 2010, 3258), tiene lugar la citación o reproducción del discurso. Como explican Cliff y Holt $(2007,2)$, se trata de un fenómeno que se ha analizado desde diferentes disciplinas y desde puntos de vista también diversos, entre ellos la gramática tradicional y la lingüística textual (Gallucci, 2014).² Las investigaciones que se han llevado a cabo sobre el tema en los últimos años se han centrado, sobre todo, en el análisis del estilo directo (ED) e indirecto (EI), que son los mecanismos de citación tradicionalmente analizados (Berenguer, 1994; Cameron, 1998; Girón Alconchel, 1998; van der Houwen, 1998, 2000; Fairclough, 1999; Benavent Payá, 2002, 2003, 2015; Camargo, 2004; Mateus, 2005; Gallucci, 2010, 2013, 2014; Sánchez, 2010; Fernández, 2011; Vargas, 2014; San Martín y Guerrero, 2013; San Martín, 2015; Estévez Rionegro, 2016; Repede, 2017).

El objetivo de este estudio es analizar qué tipos de enunciados de DR se reconocen en una muestra del español venezolano y, con especial énfasis en la cita directa, describir de qué manera estas distintas formas de DR se relacionan como un continuum. Para tal fin, tomaremos en cuenta tanto las características pragmáticas del tipo de entrevistas analizadas -conversaciones semidirigidas- como una serie de variables inherentes a una muestra socialmente estratificada.

\section{DEFINICIÓN DEL DR Y ALGUNAS PRECISIONES}

En primer lugar, debemos destacar que el DR es, en consonancia con el carácter reflexivo del lenguaje, una estructura textual o discursiva de complejidad variable que surge como "resultado de un acto de enunciación de carácter citativo" (Méndez García de Paredes, 2009, 483). Esto permite que dentro del DR se integren elementos discursivos procedentes de actos de enunciación ajenos efectiva y realmente proferidos, o bien recreados e imaginados por el propio acto de enunciación.

Por su propia naturaleza el DR suele aunar en su estructura dos componentes discursivos: un componente contextualizador del acto de enunciación referido y la cita propiamente dicha:

Al citar, el hablante convoca en su propio discurso estructuras lingüísticas que tienen como referencia actos de habla proferidos por otro hablante en el marco de una situación enunciativa diferente [...] En cada proceso de citación, el hablante, además de intervenir sobre un material discursivo y textual ajeno, lleva a cabo, también, una transformación en el mensaje citado de suma importancia: el contenido al que remite la cita pierde su vinculación con lo real del mundo, para adquirir su referencia en otro discurso [...] Se trata, por tanto, de una operación discursiva en la que un sujeto locutor que reproduce (Lr) propone a un interlocutor (Ir) un decir mediatizado cuya referencia se ancla en otra situación de enunciación totalmente diferente: la que un locutor originario (Lo) mantiene con otro interlocutor originario (Io) al que le propone cierto estado de cosas (Méndez García de Paredes, 2009, 483-484).

A simple vista, la inserción de una situación de enunciación en otra y las relaciones intersubjetivas que se producen en el discurso podrían considerarse una tarea con pocas dificultades, como explican García Negroni y Tordesillas (2001, 162). Sin embargo, como señalan las mismas autoras, esto es apenas "la parte visible de una gran cantidad de fenómenos lingüísticos que provienen de una problemática más general, la de la heterogeneidad” del discurso, es decir, "el encuentro en la misma unidad discursiva de elementos originarios de 
diferentes fuentes de enunciación" (2001, 162). Por esta razón es necesario estudiar las distintas posibilidades que ofrece el sistema de la lengua para “inscribir varias 'voces' en la misma enunciación, las diferentes escenas enunciativas que se generan, la responsabilidad del hablante con su discurso" y los contextos en los que tiene lugar (2001, 162-163).

\section{EL DR COMO CATEGORÍA LINGÜÍSTICA Y CONTINUUM}

Tomando como punto de partida el planteamiento de Girón Alconchel (2006), en este trabajo hemos considerado el DR como categoría lingüística en la que se integran enunciados pluriformes de DR, en virtud de que

Entre el discurso directo (DD) -tradicionalmente identificado con un esquema paratáctico- y el discurso indirecto (DI) identificado, a su vez, con el esquema de subordinación de la completiva de objeto directo (OD) - se descubre una serie de variantes intermedias que corresponden a determinados esquemas hipotácticos y a otros que son variantes menos prototípicas de la parataxis y de la subordinación prototípicas. En su conjunto todas estas formas y variantes del DR integran también un continuum que nos permite hablar de la categoría lingüística de la reproducción o del discurso referido (Girón Alconchel, 2006, 395).

Considerar el DR como categoría en la que se integran como un continuum diferentes formas de representar y/o reproducir el discurso (Tannen, 1989; Girón Alconchel, 1988, 2006; Semino, Short y Culpeper, 1997; Méndez García de Paredes, 1999, 2009; Fludernik, 2013) supone ir más allá de las formas de citación tradicionalmente consideradas (ED o DD, EI o DI) restringidas sobre todo a la escritura. En esta investigación esa escala o continuum que describiremos más adelante no está compuesta por categorías rígidas e incluye desde la reproducción de citas tradicionalmente definidas como "literales" hasta la referencia general a otros actos de comunicación.

\section{Muestra}

Para llevar a cabo esta investigación, hemos seleccionado una muestra de 16 hablantes del Corpus sociolingüístico de Caracas 2004-2013 -o PRESEEA-CA-estratificados en cuotas de afijación uniforme según edad, sexo y grado de instrucción, como puede observarse en la tabla 1:3

\begin{tabular}{|c|c|c|c|c|c|}
\hline Grupo generacional & \multicolumn{2}{|c|}{ 20-34 años } & \multicolumn{2}{|c|}{55 años y + } & Total \\
\hline Instrucción/Sexo & Hombres & Mujeres & Hombres & Mujeres & \\
\hline Grado 1 & 2 & 2 & 2 & 2 & 8 \\
\hline Grado 3 & 2 & 2 & 2 & 2 & 8 \\
\hline Total & 4 & 4 & 4 & 4 & 16 \\
\hline & \multicolumn{2}{|c|}{8} & \multicolumn{2}{|c|}{8} \\
\hline
\end{tabular}

Tabla 1. Distribución por cuotas de los hablantes de la muestra 


\section{FORMAS DEL DR SOMETIDAS A ESTUDIO Y SUS VARIANTES}

Siguiendo el planteamiento de Tognini-Bonelli (2004), en el análisis de las distintas formas del DR que presentaremos hemos adoptado una combinación del enfoque guiado por el corpus y del enfoque basado en el corpus. Según el primero, las formas escogidas son producto de la evidencia que arroja el propio corpus; en nuestro caso, datos reales que se producen en situaciones también reales de comunicación. En cambio, en el segundo, el corpus se emplea para explicar categorías ya existentes o predeterminadas, bien sea en gramáticas, estudios previos, etc. Hemos identificado seis tipos de citas -que hemos denominado propias o propiamente de DR-y dos periféricas -o impropias-. Hemos denominado a este segundo tipo de citas como impropias porque, aunque también en ellas se manifiesta la reflexividad del lenguaje, ello sucede en menor medida que en las anteriores, es decir, a diferencia de las primeras, que reproducen información y especifican el contenido citado, las citas impropias refieren un discurso, pero no lo reproducen.

\subsection{Citas propias}

Las citas que hemos clasificado como propias son las que más usan los hablantes de la muestra. Constituyen casos claros de heterogeneidad mostrada (Authier-Revuz, 1984) y, como ya hemos comentado, se caracterizan porque en su interior siempre hay reproducción, bien sea de naturaleza verbal (palabras, interjecciones, onomatopeyas) o gestual.

En este grupo incluimos el discurso directo, el discurso indirecto, la cita mixta, la cita de paralenguaje, la cita de gestos y los ecos. En los apartados que siguen nos detendremos en cada uno de estos tipos de citas.

\subsubsection{Discurso directo (DD)}

Según los datos extraídos de nuestras muestras, en entrevistas semidirigidas orales la cita directa puede manifestarse al menos de seis formas -o a través de seis marcos introductores distintos-: 4

a) Discurso directo tradicional (DDT), es decir, aquel introducido a través de un verbo conjugado que suele ser de comunicación (decir, preguntar, responder, etc.), como en (1):

(1) yo le dije $<$ cita $>$ iAy! / ¿será que mañana podemos ir? </cita> (CARA_M33_107)5

b) Discurso directo libre (DDL) o sin marco introductor (freestanding quotation, cf. Cameron, 1998):

(2) y mi papá lo fue a buscar $\varnothing /<$ cita $>$ No / yo sé dónde está él $</$ cita $>$ / él no está muy lejos $<$ cita $>$ (CARA_H31_029)

c) Discurso directo con sintagma nominal (DDSN) o $(y)+$ SN cuyo núcleo suele ser un pronombre personal, como en (3), o un sustantivo, como en (4): ${ }^{6}$ 
(3) $\quad$ y yo / $<$ cita $>$ no $<$ alargamiento/ $>$ / pinten ustedes $</$ cita $>$ / porque uno ya se está como cansando (CARA_H31_029)

(4) y entonces con ese piquete y ese rollo y mis hijas también <cita> ino! esa es mi mamá que está con ese poco de libros ahí </cita > (CARA_H33_101)

d) Discurso directo introducido por un marcador discursivo (DDM), como en (5):

(5) $\quad$ entonces $/<$ cita $>$ ċmi tío dónde está? </cita > (CARA_H33_101)7

e) Discurso directo introducido a través de $y+q u e$ (DDQ):

(6) Soy la niña santa que va visitar a su abuela / y que <cita $>$ hola / abuela </cita> (CARA_M13_083)

f) Discurso directo introducido por el adverbio así (DDA):

(7) ahora las muchachas se van con los hombres así<alargamiento/> / <cita > ivámonos! / nos queremos vivir / iy listo! </cita> (CARA_H31_029)

\subsubsection{Discurso indirecto (DI)}

En nuestras muestras, el discurso indirecto se ha manifestado mediante tres formas o variantes:

a) Discurso indirecto tradicional (DIT), es decir, aquel introducido mediante un verbo de comunicación conjugado seguido por la conjunción que, ${ }^{8}$ como en (8):

(8) Me dijeron que era la bruja bonita (CARA_M13_o83)

b) Discurso indirecto libre (DIL) o sin marco, como en (9):

(9) él me ayudaba<alargamiento/ $>$ / me<alargamiento/ $/ / \varnothing$ que me tranquilizara / Ø que pujara / y yo pujaba / (CARA_M11_012)

En este punto es fundamental aclarar que en las conversaciones que analizamos el DIL no funciona de la misma manera que en la literatura. Mientras que en esta última el DIL se caracteriza casi siempre por la confluencia de los puntos de vista del narrador de la historia y del personaje, en nuestro caso el requisito fundamental para clasificar un enunciado citativo como DIL es que estuviera estructurado como una cita indirecta, pero sin el elemento introductor prototípico del DI, es decir, un verbo de habla. En (9) nótese que después de la pausa podríamos incluir el verbo decir en pretérito imperfecto (me decía que me tranquilizara, me decía que pujara...).

c) Discurso cuasi indirecto (DCI) o estilo indirecto encubierto, que consiste en la reformulación del discurso propio o de otros interlocutores a través de una expresión citativa como según): 9 
(10) bueno / según mi sobrinita es porque soy muy divertido (CARA_H13_078)

También hemos incluido en esta categoría refranes, dichos y proverbios, como (11), aunque no suelen ser muy frecuentes en interacciones orales como las que hemos estudiado.

(11) todavía tengo ese trabajito ahí / tengo esa papa peladita / gracias a Dios / <risas = "E1"/> como dice el dicho / (CARA_H11_oo6)

\subsubsection{Cita mixta $(\mathrm{CM})^{10}$}

En la cita mixta se combinan el discurso directo (DDT) e indirecto tradicionales (DIT), como ocurre en (12):

(12) ella se echó pa' atrás y dijo que / que <cita $>$ iay no! $</$ cita $>$ porque le hacía daño / $<$ transcripción_dudosa> porque el asma / el frío (CARA_H33_102)

\subsubsection{Cita de paralenguaje (CP)}

Se trata de aquellas citas que, además de no estar introducidas por un verbo de comunicación, no representan propiamente "una acción lingüística, sino una onomatopeya o un ruidito, referencial o no, que ilustra por aproximación" un tipo de sonido u otros elementos paralingüísticos (Camargo, 2008, 7) y que también funcionan como demostraciones (Clark y Gerrig, 1990). ${ }^{11}$ Aquí hemos incluido casos como (13) y (14):

(13) veo que la gente se está devolviendo / pero así / ta ta ta ta ta (CARA_M31_036)

(14) lo importante es si se produce o no se produce y el área donde se produce / cómo se consume / cómo se distribuye / esto / < cita > pa / pa / pa </cita> (CARA_H33_101)

\subsubsection{Cita de gestos (CG)}

Las citas o reproducciones de gestos, como las de paralenguaje, "son también demostraciones en las que se representa una acción no verbal de tipo quinésico, pasada, posible o imaginaria" (Camargo, 2008, 8) como en (15) y (16). Por lo general, suelen estar introducidas por el verbo hacer seguido de así o solamente por este último adverbio.

(15) entonces el tipo hacía así ¿̇no? <observación_complementaria = "se toca la cintura y se sube la camisa" $/>$ (CARA_H33_101)

(16) iah! ino! y en las noches / imagínate / en las noches me acuesto así $<$ observación_complementaria = "mira hacia el cielo"/ > a ver las estrellas fugaces y a identificar estrellas (CARA_M33_107)

\subsection{6. $\operatorname{Ecos}(\mathrm{E})$}

Los ecos son una forma de repetición propia de la lengua hablada. Se trata de una representación mimética a través de la cual el hablante repite una estructura lingüística, 
emitida por otro interlocutor, inmediatamente después de que esta ha tenido lugar. De ahí que sean reacciones inmediatas a un texto previo y generalmente oral (cf. Fludernik, 1993), como en (17) y (18):

(17) E2: ¿qué es lo que más te gusta del Madrid? /

I: ¿̇del Madrid? / cuando jugaba en equipo (CARA_H13_077)

(18) E1: entonces / I. / háblanos un poco de tu familia /

I: ¿de mi familia? bueno / eeh los miembros más cercanos son / somos cuatro (CARA_M13_083)

\subsection{Citas impropias}

Como hemos señalado antes, las citas impropias, más que reproducir un discurso, lo refieren. A través de las citas impropias se pone de manifiesto que un acto de comunicación ha tenido lugar, pero no se especifica lo dicho. A pesar de esto, constituyen manifestaciones del DR en un sentido amplio del término. Hemos incluido en este grupo el discurso narrativizado y la cita abstracta.

\subsubsection{Discurso narrativizado (DN)}

El discurso narrativizado (DN) consiste en la "referencia simple a un acto de discurso propio o de otros interlocutores emitido en situaciones de enunciación diferentes a la actual" (San Martín y Guerrero, 2013, 265). En estos casos, un verbo de comunicación suele funcionar como "elemento sintetizador de la cita" (San Martín y Guerrero, 2013, 265), como en (19) y (20) o, en su defecto, de una acción verbal, como en (21):

(19) le dije a mi esposo y le di la clave / (CARA_M11_o11)

(20) a los profesores en el salón te / tú le preguntas algo y te lanzan un borrador / (CARA_M13_083)

(21) nosotras veníamos conversando (CARA_M11_011)

\subsubsection{Cita abstracta (CA) ${ }^{12}$}

Las citas abstractas también son metarrepresentaciones que se construyen a partir de otras representaciones (Wilson, 2000; Camargo, 2008). A diferencia de las citas propias, que se circunscriben a la esfera de lo público, estas son abstractas por su carácter de representación de "tipo lingüístico, lógico o conceptual" (Camargo, 2008, 5). En la CA, como apunta Camargo $(2008,5)$, "no hay atribución de un acto de habla o enunciado a una fuente", sino "referencias al código lingüístico o a la estructura del mismo", así como expresión de "relaciones lógicas o conceptuales" mediante el lenguaje. En estos casos, "se cita, en cierto sentido, la lengua misma, una instancia del código, manteniéndola en estado virtual. Se menciona la lengua” (Reyes, 1984, 67), como en (22): 
(22) y sale la palabra $/<$ lengua = "inglés" $>$ egg $</$ lengua $>$ para que ellos vayan asociando (CARA_M13_083)

En la tabla 2 presentamos de forma resumida la clasificación de citas propias e impropias que hemos descrito antes.

\begin{tabular}{|l|l|}
\hline \multicolumn{1}{|c|}{ Propias } & \multicolumn{1}{|c|}{ Impropias } \\
\hline i) Discurso directo & \\
a. Discurso directo tradicional (DDT) & \\
b. Discurso directo libre (DDL) o sin marco & i) Discurso narrativizado (DN) \\
c. Discurso directo con sintagma nominal (DDSN) & \\
d. Discurso directo con marcador (DDM) & \\
e. Discurso directo con y que (DDQ) & \\
f. Discurso directo con el adverbio así (DDA) & ii) Cita abstracta (CA) \\
\hline ii) Discurso indirecto & \\
a. Discurso indirecto tradicional (DIT) & \\
b. Discurso indirecto libre (DIL) o sin marco & \\
c. Discurso cuasi indirecto (DCI) & \\
\hline iii) Cita mixta (CM) & \\
\hline iv) Cita de paralenguaje (CP) & \\
\hline v) Cita de gestos (CG) & \\
\hline vi) Ecos (E) & \\
\hline
\end{tabular}

Tabla 2. Clasificación de las citas

\subsection{Tipología escalar del DR}

A lo largo de ese trabajo, hemos venido apuntando que el DR se materializa a través de una serie de fenómenos que funcionan como un continuum y que, por tanto, se organizan en torno a una escala. Diversos autores (Méndez García de Paredes, 1999; San Martín y Guerrero, 2013; Repede, 2015) han señalado ya que uno de los límites de ese continuum sería el discurso directo sin verbo introductor y el otro, la "narración de un acto de habla especificando su contenido en términos muy generales" (el DN) (Repede, 2015, 79).

San Martín y Guerrero (2013) y San Martín $(2015,227)$ vinculan las variantes del DR con su "grado de reformulación gramatical y con el efecto pragmático de las secuencias" discursivas en las que aparecen, "en términos de la relativa vivacidad o realismo de la cita", y "con las categorías de implicación (involvement) y distanciamiento (detachment)" señaladas por Chafe (1980, 1982) (San Martín y Guerrero, 2013, 266). San Martín (2015, 228) también apunta que, en términos de la teoría de los actos de habla de Searle (1969), todas las variantes del DR que forman parte de la escala mantienen la misma fuerza ilocutiva (es decir, la intención), pues lo que se modifica son los efectos perlocutivos, según el lugar que ocupa cada tipo de DR en la escala. A juicio de San Martín y Guerrero $(2013,266)$, esta escala del DR se organiza entonces "desde el menor nivel de reformulación y distanciamiento y mayor grado de realismo e implicación" (en nuestro caso, DDL, DIL, CP y E), "hasta el mayor nivel de reformulación y distanciamiento y menor grado de realismo e implicación" característico del empleo del DN y la CA.

En consonancia con la propuesta de San Martín (2015, 229), hemos utilizado una escala con diferentes puntos para describir la variedad de formas del DR que encontramos en nuestra 
muestra. Cada uno de los tipos de DR descritos puede aparecer separadamente o, como ocurre en los diálogos reconstruidos, se pueden combinar de manera secuencial en los relatos. En la figura 1 mostramos qué posición ocupan los tipos de citas propias e impropias que explicamos en la sección anterior en función de, por un lado, el nivel de reformulación y distancia enunciativa; $y$, por otro, de realismo e implicación. ${ }^{13}$

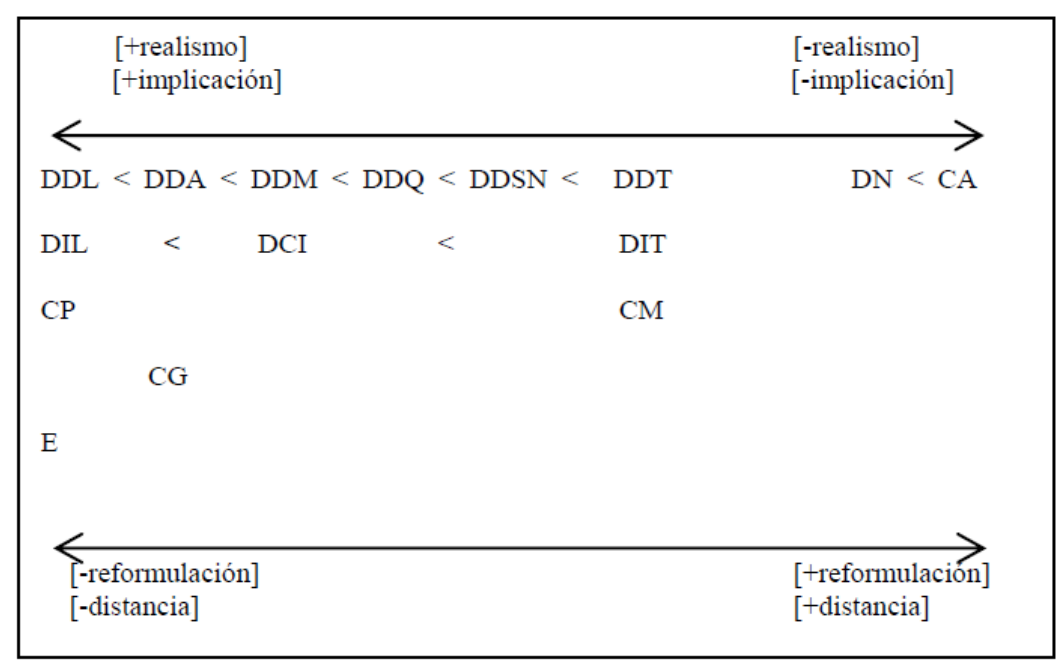

Figura 1. Tipología escalar del DR

La parte izquierda de la figura 1 muestra las citas propias. En la primera fila de esta parte se sitúan las distintas variantes del DD (DDL, DDA, DDM, DDQ, DDSN y DDT). En la segunda, las del DI (DIL, DCI y DIT), y en la tercera, la cuarta y la quinta, las CP, las CG y los E, respectivamente. Si bien en cada fila del DD y el DI ofrecemos la relación entre los tipos de cita de cada uno de estos grupos en función del grado de elaboración -o de complejidad sintáctica-, consideramos que aquellas que hemos ubicado en la misma columna, como veremos enseguida, comparten, en cierta medida, las propiedades o los rasgos que hemos identificado con los símbolos + y -. Por ejemplo: i) DDL, DIL, CP y E; ii) DDA y CG; iii) DDM y DCI; y iv) DDT, DIT y CM. La parte derecha de la escala está reservada para las citas impropias, es decir, el DN y la CA.

Como podemos apreciar en la figura 1, en su mayoría, las citas propias se ubican en la parte izquierda y en el centro de la escala, es decir, suponen más realismo e implicación y menos reformulación discursiva y distancia enunciativa. También es posible observar, como ya hemos apuntado, que hemos ubicado en la misma columna de la escala al DDL, DIL, CP y E, pues estos procedimientos funcionan de manera similar: carecen de marco introductor y, por ello, su uso supone realismo e implicación (y, también, vivacidad) en las narraciones en las que se utilizan. Por oposición, estas condiciones hacen que se traduzcan en [-reformulación], es decir, el hablante se limita a transmitir enunciados sin alterar el mensaje original, al menos en apariencia, sobre todo cuando se trata del DDL, del DIL y la CP, pues en los E sí es posible cotejar qué dice el entrevistador y qué reporta exactamente el entrevistado.

Nos detendremos ahora en el funcionamiento de las citas que se incluyen en cada grupo siguiendo el orden de presentación en la escala. Primero detallaremos los distintos tipos de citas propias y, después, las impropias. 
En cuanto a las distintas manifestaciones de la cita directa (lado izquierdo, primera fila), las presentamos en la escala a partir del DDL, es decir, la cita directa sin marco; pasando por los distintos tipos (DDA, DDM, DDQ, DDSN) que hemos ordenado, según el marco introductor en cuestión, de menor a mayor en función de la complejidad sintáctica de los elementos que lo conforman (adverbio así, marcador, $y+q u e,(y)+\mathrm{SN}$, respectivamente); para llegar finalmente a la cita directa tradicional (DDT). El DDT es el polo opuesto del DDL en el grado de contextualización y supone, a partir de la elección de un verbo introductor que suele ir conjugado en un tiempo verbal, con persona y número, [+reformulación] por parte del locutor.

Por su parte, en los tipos de cita indirecta (lado izquierdo, segunda fila), salvando las diferencias individuales de estos procedimientos frente al DD, que vienen dadas fundamentalmente por los mecanismos de transposición inherentes al DIT (deixis, etc.), hemos situado en la misma columna el DIL y el DDL, que comparten el hecho de no tener marco introductor, así como el DIT y el DDT, que sí lo tienen. En cambio, el DCI lo hemos ubicado en la columna del DDM, pues bien sea que se trate de una cita introducida a través de según o de un dicho, consideramos que funcionan de manera análoga a los marcadores del discurso como introductores de cita.

En la tercera fila hemos ubicado, por un lado, la CP y, por otro, la CM. Hemos considerado la cita de paralenguaje en la misma columna del DDL y el DIL, pues suele aparecer, como estos últimos, sin marco introductor. Por su parte, la cita mixta, al ser un mecanismo de citación híbrido, o sea, a medio camino entre el DDT y el DIT, la hemos ubicado en la misma columna que estos últimos en virtud de las propiedades que comparten.

En la cuarta fila hemos ubicado la cita gestual. La hemos alineado con el DDA, ya que en nuestra muestra la CG suele ir introducida, como aquella, a través del adverbio así. Por último, en la quinta línea de la figura 1 hemos puesto los ecos. Los E, como el DDL, el DIL y la CP, carecen de marco introductor. Por ello se traducen en [-reformulación] y [-distancia].

En la parte derecha de la escala, identificada con [-realismo], [-implicación], [+reformulación] y [+distancia], se agrupan las citas impropias, ya que se trata del polo opuesto de los procedimientos citativos que hemos descrito antes. Tanto el DN como la CA suponen más reformulación discursiva, pues el locutor debe emplear una expresión que sintetice un acto verbal en lugar de especificar qué se dijo realmente, como sí suele ocurrir en las citas propias que se caracterizan, precisamente, por expresar el contenido citado. Esto supone que las citas impropias -es decir, el DN y la CA-14 se traducen en menos realismo frente a lo que se está contando y, en consecuencia, el relato en cuestión suele perder vivacidad e histrionismo. A diferencia de San Martín y Guerrero (2013), nuestro límite de la escala lo constituyen las citas abstractas (CA), que en nuestro caso van antecedidas por el DN.

\section{RESULTADOS Y ANÁLISIS}

\subsection{Citas propias e impropias}

En primer lugar, daremos cuenta del uso de los dos tipos generales de DR que en esta investigación hemos identificado bajo las etiquetas de citas propias e impropias. En la tabla 3 observamos el total de citas que obtuvimos de la muestra de habla del español caraqueño -2.094 casos- desglosado en función de esta primera clasificación general. 


\begin{tabular}{|l|r|r|}
\hline Tipo de cita & $\mathrm{N}$ & $\%$ \\
\hline Propia & 1.564 & 74,7 \\
\hline Impropia & 530 & 25,3 \\
\hline Total & 2.094 & 100 \\
\hline
\end{tabular}

Tabla 3. Tipos generales de cita identificados en la investigación

Como se aprecia en la tabla $3,1.564$ casos $(74,7 \%$ del total) corresponden a citas propias -o tradicionales- y 530 (25,3\%) a citas impropias. En la tabla 4 presentamos la distribución de las citas propias en función de los tipos específicos que ya comentamos.

\begin{tabular}{|l|r|r|}
\hline Citas propias & $\mathrm{N}$ & $\%$ \\
\hline $\mathrm{DD}$ & 1.205 & 77 \\
\hline $\mathrm{DI}$ & 261 & 16,7 \\
\hline $\mathrm{CP}$ & 34 & 2,2 \\
\hline $\mathrm{CG}$ & 28 & 1,8 \\
\hline $\mathrm{CM}$ & 19 & 1,2 \\
\hline $\mathrm{E}$ & 17 & 1,1 \\
\hline Total & 1.564 & 100 \\
\hline
\end{tabular}

Tabla 4. Distribución de las citas propias

La tabla 4 dejar ver la preferencia de los hablantes caraqueños por la cita directa. De los 1.564 casos de citas propias, 1.205 casos (77\%) son de DD. A este último le siguen en orden decreciente, pero muy por debajo, los casos de DI con 261 ocurrencias, que constituyen apenas el $16,7 \%$, y, seguidamente, las citas de paralenguaje $(2,2 \%)$, la cita de gestos $(1,8 \%)$, la cita mixta $(1,2 \%)$ y los ecos (1,1\%). Si bien es cierto que estos últimos mecanismos del DR se usan poco, al menos en nuestra muestra, pues en conjunto no superan el $6 \%$ de los enunciados citativos, forman parte del abanico de posibilidades del hablante para reproducir -e imitar- (cf. Clark y Gerrig, 1990) otros discursos.

En la tabla 5 ofrecemos la distribución de las citas impropias en nuestra muestra del español caraqueño.

\begin{tabular}{|l|r|r|}
\hline Citas impropias & $\mathrm{N}$ & $\%$ \\
\hline DN & 519 & 97,9 \\
\hline CA & 11 & 2,1 \\
\hline Total & 530 & 100 \\
\hline
\end{tabular}

Tabla 5. Distribución de las citas impropias

La tabla 5 muestra que las citas impropias suelen ser casi exclusivamente de discurso narrativizado, procedimiento que ronda el 97,9\% de los casos (519 de un total de 530). El 2,1\% restante (apenas 11 enunciados) corresponde a las citas abstractas. 
En el gráfico 1 ofrecemos en conjunto la distribución de los 2.094 casos encontrados en función de los tipos específicos de citas propias e impropias -8 en total- que hemos identificado en nuestra investigación.

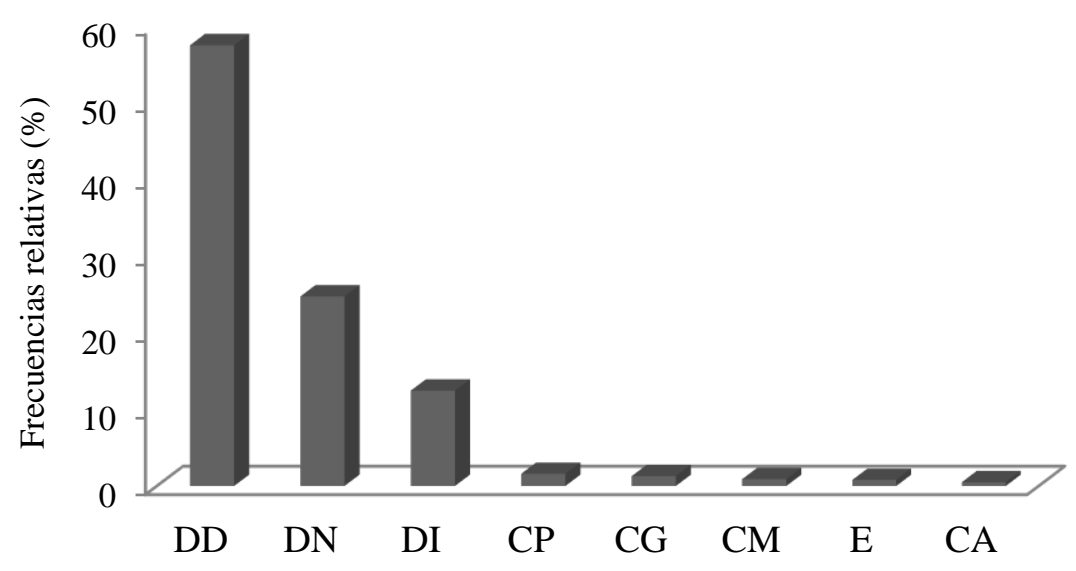

Gráfico 1. Distribución del DR en la muestra

Como se desprende del gráfico 1, cuando unimos los fenómenos que forman parte de las citas propias e impropias, el procedimiento del DR más empleado sigue siendo la cita directa, con 1.205 ocurrencias, que representan el 57,5\% del total, esta vez seguido del discurso narrativizado, con 519 casos (24,8\%), y de la cita indirecta, con 261 (12,5\%). Estos tres tipos de DR conforman el 94,8\% de los enunciados citativos de la muestra analizada. El resto tiene menos de 40 casos y en conjunto constituye, como ya hemos visto, el $5,1 \%$ del total de ocurrencias.

La preferencia por la cita directa en español también se observa en todas las investigaciones que constituyen los antecedentes de este trabajo en lo que a análisis de muestras orales se refiere, tanto del español de América como del de España (Berenguer, 1994; Cameron, 1998; Girón Alconchel, 1998; van der Houwen, 1998, 2000; Fairclough, 1999; Benavent Payá, 2002, 2003, 2015; Camargo, 2004; Mateus, 2005; Gallucci, 2010; Sánchez, 2010; Fernández, 2011; Vargas, 2014; San Martín y Guerrero, 2013; San Martín, 2015; Estévez Rionegro, 2016; Repede, 2017). En todos estos estudios, la frecuencia de DD suele ser muy superior al 50\% del total de casos analizados, independientemente del tamaño de la muestra.

En la presente investigación, como ya hemos visto, si tomamos el DD en el grupo de las citas propias, representará un $77 \%$ de los casos. En cambio, si lo vemos en conjunto, es decir, con todos los otros tipos de citas -tanto propias como impropias- constituye el 57,5\% del total de ocurrencias, en virtud del alto número de casos de discurso narrativizado presente en nuestras entrevistas.

\subsection{Variantes de las citas propias: el DD}

Una vez que hemos comentado los resultados de los tipos generales del DR, se hace necesario indagar en sus variantes. Por tratarse del procedimiento citativo que constituye más de la mitad de los casos que encontramos y dadas las dimensiones de este artículo, nos limitaremos solamente a comentar los distintos tipos de DD. 
En la tabla 6 ofrecemos la distribución de las distintas variantes de la cita directa.

\begin{tabular}{|l|r|r|}
\hline Tipo de DD & $\mathrm{N}$ & $\%$ \\
\hline DDL & 502 & 41,7 \\
\hline DDT & 490 & 40,7 \\
\hline DDSN & 141 & 11,7 \\
\hline DDM & 44 & 3,7 \\
\hline DDQ & 24 & 2,0 \\
\hline DDA & 4 & 0,3 \\
\hline Total & 1.205 & 100 \\
\hline
\end{tabular}

Tabla 6. Distribución del DD y sus variantes

Los datos de la tabla 6 evidencian que, si bien tradicionalmente se ha considerado que el DD suele estar introducido, sobre todo, por un verbo, como anuncian las gramáticas del español (cf. Gallucci 2012, 2017), las citas directas introducidas $\varnothing$ verbo -o sea, el DDL- superan, aunque por poco, a las primeras: 502 casos de DDL $(41,7 \%)$ y $490(40,7 \%)$ de DDT. Este margen de diferencia es pequeño -apenas 1\%- pero fundamental, pues si nos restringimos a lo apuntado en las gramáticas en este sentido, esto limitaría el alcance real de este procedimiento de cita en la conversación, y este ha sido precisamente uno de los objetivos de la investigación de mayor alcance en la que se enmarca este artículo.

En consonancia con el propósito de este trabajo, como ya hemos señalado, nos referiremos a cada uno de los tipos de DD que identificamos en las muestras de habla del PRESEEA-CA.

En nuestras entrevistas, el DDL o zero quotative (cf. Mathis y Yule, 1994) suele aparecer sobre todo en un tipo de contexto específico: el de los diálogos reconstruidos (Tannen, 1986, 1989), como en (23). En efecto, de los 502 casos de DDL, 314 forman parte de uno de estos diálogos.

(23) cita> oye / mira / hay lo siguiente / ¿̇por qué estás cansado? </cita > < cita > profesor / yo estoy cansado porque usted / sabe $\mathrm{pa}^{\prime}$ / $\mathrm{pa}^{\prime}$ lidiar con malandros y eso es muy difícil / yo quiero que usted me ayude para pasar Historia Universal / sabe que me tiene que pasar $</$ cita $>$ / <cita $>$ bueno / vamos a hacer lo siguiente / tú presentas tu prueba / estudias / si sacas un nueve te pongo diez $</$ cita $>$ / $<$ cita $>$ ihecho / profe! $<$ /cita $>$ (CARA_H33_101)

La cita directa tradicional -o DDT- también puede formar parte de un diálogo reconstruido (DREC), aunque en menor proporción (185/490). En nuestras muestras, el DDT se ha materializado a través de 62 formas verbales distintas que incluyen verbos que no son de comunicación sino de atribución, como ser, o de desplazamiento, como llegar, venir, etc. A continuación ofrecemos el listado completo de formas verbales del DDT presentes en nuestras entrevistas:

acabar; acercarse; advertir; agarrar; andar; apoyar; avisar; burlarse; comentar; contar; convencer; decir; empezar; empezar a + infinitivo (avisar, gritar, hablar); escribir; escuchar; estar; estar + gerundio (acordándose, diciendo, hablando, peleando); gritar; hablar; invitar; 
ir a + infinitivo (decir, empezar); ir + gerundio (corrigiendo, diciendo, llegando); llamar; llegar; llegar a decir; mandar; mantenerse; molestarse; orar; participar; pegar (el grito en el cielo); pelear; poder + infinitivo (decir, poner); ponerse; ponerse a + infinitivo (cantar, inventar); preguntar; proponer; quejarse; salir; saludar; ser; silbar; subir; tener que + infinitivo (estar); venir, ver, volver a venir.

De los 490 casos de DDT, 375 se introducen con decir, "el más usual e incoloro de los verbos que introducen estilo directo" (cf. Alonso, 1973, 196). Este resultado también coincide con todos los antecedentes de nuestro estudio. A este verbo le siguen, con frecuencias absolutas muy inferiores, llegar, ser, llamar, preguntar y venir con 10, 9, 8 y 7 casos, respectivamente. $\mathrm{El}$ resto de las formas que detallamos en la lista anterior registra menos de 5 casos en toda la muestra.

Por su parte, del discurso directo con sintagma nominal (DDSN) en las entrevistas se registran 141 casos. De este total, 111 corresponden a un pronombre y 30 a un nombre. Como es bien sabido, frente al sustantivo el pronombre se enmarca dentro de los mecanismos de economía del lenguaje.

Del DDM, es decir, de la cita directa introducida por un marcador discursivo, hemos encontrado 44 registros. Los marcadores que utilizan los hablantes de la muestra en sus narraciones antes de una cita directa son cuatro: bueno, de repente, entonces y o sea. Como se desprende del gráfico 2, el que más se usa en nuestra muestra es entonces.

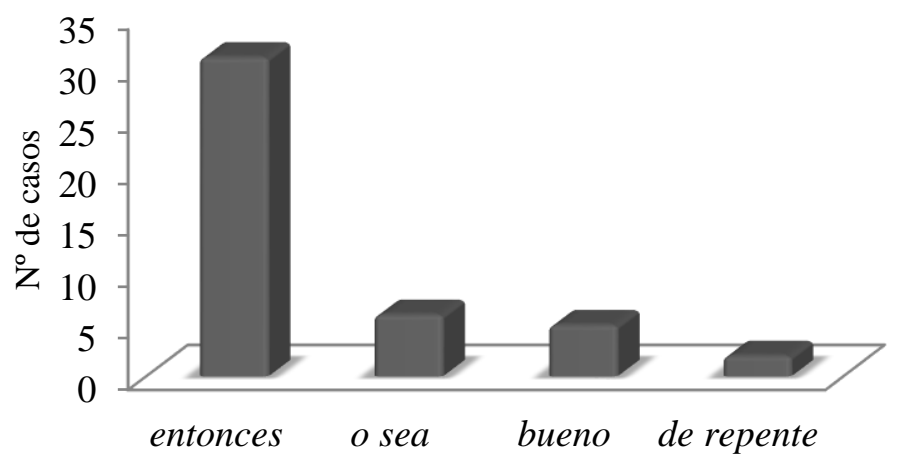

Gráfico 2. Marcadores introductores de DDM

En lo que respecta al DDQ, en nuestro corpus se registran 24 ocurrencias que representan el 2,2\% del total de DD -1.205-. De esos 24 ejemplos, 15 forman parte de un diálogo reconstruido, como en (24), y cumplen una función narrativa, aunque formen parte de una secuencia dialogal. En cambio, los que no forman parte de un DREC, como en (25), suelen formar parte de una secuencia descriptiva y sirven para ofrecer un ejemplo.

(24) no<alargamiento/> itenían / tenían como un año! / idos años! / y que <cita $>$ ison novios / pana! </cita > o sea yo le dije a M. <cita > ćcómo / cómo puede ser su novio? $</$ cita $>$ entonces < cita $>$ verga / no sé y broma </cita $>$ / entonces / M. eeh / eeh / M. E. C le pregunta a M. <cita $>$ ay / ¿y por qué tú no trajiste a tu novia? $</$ cita $>/<$ cita $>$ porque pa' la playa no se lleva arena $</$ cita $>$ le dice M. / <risas = "I"/ $>$ entonces M. E. C. $<$ cita $>$ ah / sí </cita $>$ al día siguiente eeh / ella le dice este <alargamiento/ $><$ cita $>$ ¿viste? / tenías razón / pa' la playa no se lleva arena </cita> (CARA_H13_077) 
(25) mira / más de una vez nosotras estamos reunidas haciendo un trabajo teórico y los hombres están haciendo la / el material / < risas = "I" / > y que < cita $>$ tú recorta foami / recorta cartulina / recorta lo que tú quieras / pero recorta y haz algo / no me fastidies ahorita $</$ cita $><$ risas = "I, E1" $/>$ pero es eso / y salimos los fines de semana / que el cine / que esto / pero sí / sí / sí es complicado / (CARA_M13_083)

Del DDA hemos encontrado apenas 4 casos (o,3\% del total), que son muy pocos para describir con propiedad alguna tendencia en este sentido.

\subsection{El DR y las variables sociales}

En lo que respecta a las variables sociales inherentes a nuestra muestra del PRESEEA-CA (edad, sexo y grado de instrucción), debemos comentar dos cuestiones. En primer lugar, a pesar de que en principio consideramos el cálculo del $\chi^{2}$ incluyendo todos los fenómenos del DR que registramos, con la finalidad de poder establecer estadísticamente si hay relación o no entre el tipo de DR que se emplea y las variables sociales independientes de nuestra muestra, decidimos prescindir de este cálculo, pues, como apunta Larson-Hall (2012, 267), no podemos aplicarlo si tenemos menos de 5 ocurrencias en alguna casilla de las tablas, como es nuestro caso.

En segundo lugar, y en virtud de lo anterior, calculamos con el programa SPSS Statistics 22.o los valores de $\chi^{2}$ y $p$ en tablas de contingencia que incluyeran solamente el DD y el DI, tipos de DR que comparten dos características ampliamente relacionadas: i) un contenido citado y ii) la función general del DR: reproducir palabras. ${ }^{15}$ Estas dos propiedades nos permiten considerarlos, en un sentido amplio, como variantes de la variable dependiente DR. Las dos variantes del DR que hemos seleccionado según lo apuntado antes (es decir, el DD y el DI) suman 1.466 ocurrencias y representan el 70\% del total de casos del DR -2.094- que encontramos en nuestras muestras. Nos limitaremos a presentar aquí las variables sociales que han resultado significativas.

En esta oportunidad, se trata solamente de la variable 'sexo' o género de los hablantes cuya distribución detallamos en la tabla 7.

\begin{tabular}{|c|c|c|c|c|}
\hline \multirow{2}{*}{\multicolumn{2}{|c|}{ Sexo }} & \multicolumn{2}{|c|}{ Discurso referido } & \multirow{3}{*}{$\begin{array}{r}\text { Total } \\
75^{2}\end{array}$} \\
\hline & & \multirow{2}{*}{$\begin{array}{r}\text { DD } \\
660\end{array}$} & \multirow{2}{*}{$\begin{array}{l}\text { DI } \\
92\end{array}$} & \\
\hline Hombres & $\mathrm{N}$ & & & \\
\hline & $\%$ & 87,8 & 12,2 & 100 \\
\hline \multirow[t]{2}{*}{ Mujeres } & $\mathrm{N}$ & 545 & 169 & 714 \\
\hline & $\%$ & 76,3 & 23,7 & 100 \\
\hline \multirow[t]{2}{*}{ Total } & $\mathrm{N}$ & 1.205 & 261 & 1.466 \\
\hline & $\%$ & 82,2 & 17,8 & 100 \\
\hline \multicolumn{2}{|c|}{$x^{2}=32,729$} & g.d.l $(3,8$ & \multicolumn{2}{|c|}{$p=0,000$} \\
\hline
\end{tabular}

Tabla 7. DD y DI y la variable sexo 
Como se aprecia en la tabla 7 , según la prueba del $\chi^{2}$, hay probabilidad de que el sexo incida en el uso variable del DR ( $p=0,000<0,050)$, ya que el valor de la muestra $(32,729)$ supera el esperado $(3,841)$. En este caso, se rechaza la Ho -o de partida- que postula que dos variables no están relacionadas. Los datos confirman la tendencia de las mujeres al uso de la cita indirecta, como ya apuntábamos en Gallucci (2010, 2013, 2014). También, un número ligeramente mayor de casos de DD en los hombres.

La comparación con algunos de nuestros antecedentes -aquellos que dan cuenta de variables sociales- deja ver que las tendencias a propósito de la influencia de la variable independiente 'sexo' en el DR son divergentes. Nuestros hallazgos a propósito de un mayor número de citas en las mujeres y, también, de más casos de DI en este grupo coinciden parcialmente con Gallucci (2013) y San Martín y Guerrero (2013), en lo que respecta a la frecuencia similar de empleo de las variantes generales de DR según el sexo de los informantes, a pesar de que estos últimos autores registran diferencias más marcadas que se traducen en un mayor porcentaje de aparición en las mujeres con un 40,8\% de DD y un 14,6\% de DI, frente al $33,5 \%$ de DD y el 11,1\% de DI en los hombres. Esta situación se repite en parte en San Martín $(2015,376)$, estudio en el que el autor encontró que "las mujeres usan más el DD $(73,1 \%)$ y los hombres el DI (33,1\%)", aunque "las mujeres emplean más el discurso referido en general". ${ }^{16}$ Lo mismo ocurre en Guerrero (2014), aunque esta variable externa no manifieste significatividad estadística en la investigación de la autora. En nuestro caso, las entrevistadas usan un poco más el DR que los hombres y han sido estos últimos los que más emplean el DD, en contraste con las mujeres, que hacen lo propio pero con el DI, como ya hemos indicado.

A diferencia de lo que acabamos de señalar, Fernández (2011) y Vargas (2014) indican que en sus muestras el sexo-género no condiciona el uso que hacen los hablantes del DR. En este punto hay que subrayar que ambas autoras analizan muy pocas muestras en las que encuentran, además, pocos casos. ${ }^{17} \mathrm{Al}$ hilo de lo anterior, pero con otro tipo de corpus, Fairclough (1999) concluye, según sus datos, que en el habla culta mexicana los hombres tienden a utilizar el DI con mayor frecuencia que las mujeres.

\section{REFLEXIÓN FINAL Y NUEVAS PERSPECTIVAS}

El estudio aquí presentado ha dejado ver la aplicabilidad del modelo de citas propias e impropias que hemos establecido en torno a una escala para dar cuenta de la heterogeneidad enunciativa (Authier-Revuz, 1984) en la narración oral conversacional. Los datos han pretendido mostrar el abanico de posibilidades que ofrece el sistema de la lengua para incorporar otras voces en nuestros discursos; también, la preferencia de un grupo de hablantes caraqueños en este sentido. Aunque gran parte de los resultados obtenidos sobre las categorías lingüísticas coinciden con los estudios previos que se han hecho sobre el DR en el español oral (uso del DD, empleo del verbo decir), además de ampliar la muestra de estudio, sería deseable llevar a cabo estudios contrastivos en comunidades de habla de otras variedades del español en los que se replique el presente trabajo, o algunos de sus aspectos.

En lo que respecta a las variables extralingüísticas que guardan relación con el DR, hemos visto que, en sentido estricto, solamente cuando se toman en cuenta el DD y el DI el $\chi^{2}$ permite interpretar que la variable 'sexo' se relaciona, al menos preliminarmente, con el uso de estos procedimientos citativos en nuestras entrevistas. Como ya hemos visto, en las distintas investigaciones sobre el tema que toman en consideración variables extralingüísticas hay debate sobre la influencia de los factores sociales en los distintos mecanismos de citación. Por 
esta razón, consideramos que en futuras investigaciones sobre el DR este tipo de controversia podría resolverse mediante el uso de nuevas herramientas estadísticas, como los modelos de efectos mixtos (mixed effect models) (Baayen, 2008; Jaeger, 2008; Tagliamonte, 2012, 2016) que incluyen al hablante como un efecto aleatorio, lo que permite incluir la variabilidad individual inherente a una muestra sociolingüísticamente estratificada.

\section{NOTAS}

1 Las etiquetas discurso citado o discurso reproducido "funcionan, grosso modo, como sinónimos de discurso referido" (Méndez García de Paredes, 2009, 483). Aunque algunos autores no están de acuerdo con esta distinción (por ejemplo, Maldonado, 1991, 20), en este trabajo lo hemos considerado de esta manera, ya que hemos estudiado tanto la reproducción de una acción realizada verbalmente como la situación de enunciación en la que tiene lugar.

2 Para una descripción del discurso referido en las gramáticas del español, en los manuales sobre análisis del discurso y pragmática lingüística, así como en los diccionarios de español, véase Gallucci (2016, 2017) y Gallucci y Ruiz (en prensa), respectivamente.

3 Sobre los orígenes, el desarrollo y el estado actual del proyecto PRESEEA consúltese, entre otros, Moreno Fernández (1997, 2004, 2005a, 2005b) y Moreno Fernández et. al (2001).

4 Si bien estas distintas formas pueden aparecer en un mismo enunciado de DR como un continuum, en el que se produce una mezcla o hibridación de las mismas, para facilitar la explicación de cada una en lo que sigue las presentaremos separadamente.

5 El código especificado al final de los ejemplos debe leerse, de izquierda a derecha, de la siguiente forma: i) CARA_= Caracas; ii) sexo o género: $\mathrm{H}=$ hombres, $\mathrm{M}=$ mujeres; iii) edad: $1=20$ a 34 años, $2=35$ a 55 años, $3=55$ años en adelante; iv) grado de instrucción: 1 = analfabetos o sin estudios, 2 = enseñanza secundaria, 3 = enseñanza superior; $v$ ) identificación del hablante dentro del corpus: 005, 006, 036, 101, etc. Por ejemplo, a través del código CARA_M33_107 se identifica a una mujer caraqueña, de más de 55 años, con estudios universitarios, identificada con el número 107 en nuestro corpus.

6 En el DDSN, la conjunción puede estar presente o no. El elemento introductor es el sintagma nominal en cuestión.

7 Aunque este uso de entonces como marco introductor de cita suele ser muy raro en el español peninsular, sí se emplea de esta manera en el español americano, al menos en la variedad caraqueña.

8 Como afirma Verdín Díaz (1970, 52-53), la conjunción que introduce la cláusula reportada en DI no siempre es que, como se recoge en varias gramáticas, aunque sí se trata de la más usada en español. $\mathrm{El}$ autor destaca que "no es necesario que la partícula indicadora de la subordinación sea una verdadera conjunción, como en el caso de El médico preguntó si María tenía fiebre, ya que si, al introducir una interrogativa indirecta, lejos de su valor de conjunción condicional, pasa a ser una partícula subordinante" (Gallucci, 2013, 102).

9 El DCI suele tratarse como una variedad del DIL por la "ausencia de señales sintácticas de subordinación (ambos comparten la ausencia de verbo de habla y subordinante y también la esporádica posposición de expresiones como según dicen”, etc.) (Reyes, 1994, 21). Nosotros hemos optado por analizarlo en el mismo grupo del DI, pero haciendo la distinción entre este y el DIL.

10 En este caso y en el de las dos categorías que siguen, nos apoyamos en la propuesta de Camargo (2008) a propósito del análisis de las citas conversacionales en el PRESEEA. 
11 De ahí que Camargo (2008) las denomine, junto a las citas de gestos, como citas no lingüísticas.

12 Otros autores la llaman cita metalingüística (Reyes, 1984), glosa (Reyes, 2002) o cita pura (Cappelen y Lepore, 2007).

13 De San Martín y Guerrero (2013) y de San Martín (2015) hemos tomado como referencia los valores generales que se consideran en la escala. La escala en sí misma, la ubicación de cada tipo de cita extraída de la muestra y la analogía entre ellas es de nuestra autoría.

14 Debemos aclarar en este punto que, si bien las citas abstractas implican, por un lado, [-realismo] y [-implicación], ciertamente es más ajustado a su funcionamiento que, por otro, se traduzcan, en el punto opuesto de la escala, en [-reformulación] y [-distancia], pues a través de ellas la posibilidad de reformulación es muy baja. Agradezco a Charlotte Coy, del Romanisches Seminar de la Eberhard Karls Universität Tübingen, esta observación.

15 Si bien es cierto que las funciones específicas del DR van más allá de la mera reproducción de palabras (narrar, ejemplificar, argumentar, etc.) (cf. Gallucci, 2010, 2013; Camargo, 2008), en esta oportunidad hemos simplificado esta cuestión.

16 Sin embargo, en este punto debemos recordar que el autor incluye en el DI lo que nosotros hemos analizado como DN.

17 Para una versión resumida de Vargas (2014), consúltese Gallucci y Vargas (2015).

\section{REFERENCIAS BIBLIOGRÁFICAS}

ALONSO, D. 1973. "El anuncio del estilo directo en el 'Poema del Cid' y en la épica francesa", en Alonso, D. (coord.), Obras completas. Estudios y ensayos sobre literatura. Vol. 2., $1^{\mathrm{a}}$ parte. "Desde los orígenes románicos hasta finales del siglo XVI". Madrid: Gredos, pp. 195-214.

AUTHIER-REVUZ, J. 1984. “Heterogéneité(s) énonciative(s)", Langages, 73, pp. 98-111.

BAAYEN, H. 2008. Analyzing linguistic data: a practical introduction to statistics using $R$. Cambridge: Cambridge University Press.

BENAVENT PAYÁ, E. 2002. "Límites entre la oralidad y la escritura: formas de 'decir' el discurso directo en los relatos de ayer y hoy", Res Diachronicae, 1, pp. 70-81.

BENAVENT PAYÁ, E. 2003. "¿Por qué contamos nuestras historias cotidianas en estilo directo?”, en Delbecque, N. (ed.), Aproximaciones cognoscitivo-funcionales al español. Ámsterdam / New York: Rodopi, pp. 11-20.

BENAVENT PAYÁ, E. 2015. Decir y discurso directo en los relatos de la conversación coloquial. Tesis doctoral. Valencia: Universitat de València.

BENTIVOGLIO, P. y MALAVER, I. 2006. "La lingüística de corpus en Venezuela: un nuevo proyecto", Lingua Americana, 19, pp. 37-46.

BENTIVOGLIO, P. y MALAVER, I. 2012. "Corpus sociolingüístico de Caracas: PRESEEA Caracas 2004-2010. Hablantes de instrucción superior”, Boletín de Lingüística, 24(37-38), pp. 144-180.

BERENGUER, J. 1994. Estrategias del discurso conversacional: algunos casos de relato coloquial en catalán y español. Tesis doctoral. Valencia: Universitat de València.

CAMARGO, L. 2004. La representación del discurso en la narración oral conversacional. Estudio sociopragmático. Tesis doctoral. Alcalá de Henares: Universidad de Alcalá.

CAMARGO, L. 2008. "La representación del discurso en los corpus de PRESEEA: metodología y propuesta de análisis". Comunicación presentada en el $X V$ Congreso Internacional de la ALFAL, 18-21 de agosto, Montevideo. 
CAMERON, R. 1998. "A variable syntax of speech, gesture, and sound effect: direct quotations in Spanish", Language Variation and Change, 10, pp. 43-83.

CAPPELEN, H. y LEPORE, E. 2007. Language turned on itself. Oxford: Oxford University Press.

CHAFE, W. (ed.). 1980. The peer stories: cognitive, cultural and linguistic aspects of narrative productions. Norwood, NJ: Ablex.

CHAFE, W. 1982. "Integration and involvement in speaking, writing and oral literature", en Tannen, D. (ed.), Spoken and written language. Exploring orality and literacy. Norwood, NJ: Ablex.

ESTÉVEZ RIONEGRO, N. 2016. Las construcciones de estilo directo en español. Estudio de corpus. Tesis doctoral. Santiago de Compostela: Universidade de Santiago de Compostela.

FAIRCLOUGH, M. 1999. "Discurso directo vs. discurso indirecto en el español hablado en Houston”, The Bilingual Review, 24(3), pp. 217-229.

FERNÁNDEZ, M. F. 2011. Uso del discurso directo e indirecto en el habla de Mérida. Tesis de grado. Mérida: Universidad de Los Andes.

FLUDERNIK, M. 2013. "Conversational narration - Oral narration”, en Hühn, P. et al. (eds.), The living handbook of narratology. Hamburgo: Hamburg University. Disponible en: http://www. lhn.uni-hamburg.de/article/conversational-narration-\%E2\%80\%93-oral-narration.

GALLUCCI, M. J. 2010. Discurso directo y discurso indirecto en el habla de Caracas. Tesis de maestría. Caracas: Universidad Central de Venezuela.

GALLUCCI, M. J. 2012. "Estilo directo e indirecto en interacciones orales. Estado de la cuestión en el ámbito hispánico", Boletín de Filología, 47(2), pp. 205-233.

GALLUCCI, M. J. 2013. "Más sobre el estilo directo e indirecto en el español de Caracas”, Lengua y Habla, 17, pp. 89-111.

GALLUCCI, M. J. 2014. "Entonces un muchacho llega y me dice: 'Mira, regáleme un bolívar'. Las citas en estilo directo e indirecto en el Corpus diacrónico del habla de Caracas 1987/2013", Boletín de Lingüística, 26(41-42), pp. 43-72.

GALLUCCI, M. J. 2016. "El discurso referido en los manuales sobre análisis del discurso y pragmática lingüística”, Lengua y Habla, 20, pp. 201-224.

GALLUCCI, M. J. 2017. "El discurso referido en la tradición gramatical hispánica", Cuadernos de Lingüística, 4(2), pp. 213-256.

GALLUCCI, M. J., GONZÁLEZ, C., y MALAVER, I. 2013. "Corpus sociolingüístico 'PRESEEA Caracas 2004-2010’. Hablantes de grado 1 de instrucción”, Boletín de Lingüística, 25(39-40), pp. 92-107.

GALLUCCI, M. J. y VARGAS, K. 2015. "Estilo directo e indirecto en el Corpus sociolingüístico de Caracas 1987”, Anuario de Letras, 3(2), pp. 65-103.

GALLUCCI, M. J. y RUIZ, S. (en prensa). "El discurso referido en los diccionarios de español", Akademos, 19(1-2).

GARCÍA NEGRONI, M. M. y TORDESILLAS, M. 2001. La enunciación en la lengua. De la deixis a la polifonía. Madrid: Gredos.

GIRÓN ALCONCHEL, J. L. 1988. "La reproducción del discurso en la lengua hablada", en Actas del II Simposio Internacional de Semiótica. Lo cotidiano y lo teatral. Vol. I. Oviedo: Servicio de Publicaciones de la Universidad de Oviedo, pp. 203-215.

GIRÓN ALCONCHEL, J. L. 2006. "Sobre la gramaticalidad del discurso referido", en Company, C. (ed.), El español en América. Diatopía, diacronía e historiografía. Homenaje a José Moreno de Alba. México, DF: Universidad Nacional Autónoma de México, pp. 395-406.

JAEGER, F. 2008. "Categorical data analysis: away from ANOVAs (transformation or not) and towards logit mixed models”, Journal of Memory and Language, 59, pp. 434-446. 
LARSON-HALL, J. 2012. "How to run statistical analyses", en Mackey, A. y Gass, S. (eds.), Research methods in second language acquisition: a practical guide. Oxford: Wiley-Blackwell, pp. 245-274.

MALDONADO, C. 1991. Discurso directo y discurso indirecto. Madrid: Taurus.

MATEUS, L. 2005. El estilo directo e indirecto como estrategias narrativas en el habla de Caracas. Trabajo de grado de licenciatura. Caracas: Universidad Central de Venezuela.

MATHIS, T. y YULE, G. 1994. "Zero quotatives”, Discourse Processes, 18(1), pp. 63-76.

MÉNDEZ GARCÍA DE PAREDES, E. 1999. "Análisis de la reproducción del discurso ajeno en los textos periodísticos”, Pragmalingüística, 7, pp. 99-128.

MÉNDEZ GARCÍA DE PAREDES, E. 2009. "Los enunciados del discurso referido y la gramática", en Camacho, M. V., Rodríguez, J. J., y Santana, J. (eds.), Estudios de lengua española: descripción, variación y uso. Homenaje a Humberto López Morales. Frankfurt am Main: Iberoamericana/Vervuert, pp. 483-517.

MORENO FERNÁNDEZ, F. 1997. "Metodología del 'Proyecto para el Estudio Sociolingüístico del Español de España y América”, en Moreno Fernández F. (ed.), Trabajos de sociolingüística hispánica. Alcalá de Henares: Universidad de Alcalá, pp. 137-161.

MORENO FERNÁNDEZ, F. 2004. "Corpora of spoken Spanish language -The representativeness issue-”, en Kawaguchi, Y., Zaima, S., Takagaki, T., Shibano, K., y Usami, M. (eds.), First International Conference on Linguistic Informatics. State of the art and the future. Tokio: University of Foreign Studies, pp. 49-76.

MORENO FERNÁNDEZ, F. 2005a. "Corpus para el estudio del español en su variación geográfica y social. El corpus 'PRESEEA”, Oralia, 8, pp. 123-139.

MORENO FERNÁNDEZ, F. 2005b. "Project for the sociolinguistic study of Spanish from Spain and America (PRESEEA) -A corpus with a grammar and discourse bias-", en Takagaki, T., Zaima, S., Tsuruga, Y., Moreno-Fernández, F., y Kawaguchi, Y. (eds.), Corpus-based approaches to sentence structures. Ámsterdam: John Benjamins, pp. 265-288.

MORENO FERNÁNDEZ, F., CESTERO MANCERA, A. M., MOLINA MARTOS, I., y PAREDES GARCÍA, F. 2001. "El Proyecto para el Estudio Sociolingüístico del Español de España y América (PRESEEA): antecedentes, objetivos y estado actual”, en Ruiz Miyares, L. (ed.), Actas del VII Simposio Internacional de Comunicación Social. Málaga: Centro de Lingüística Aplicada / Universidad de Málaga, pp. 45-47.

NIEMELÄ, M. 2010. "The reporting space in conversational storytelling: orchestrating all semiotic channels for taking a stance”, Journal of Pragmatics, 42(12), pp. 3258-3270.

REPEDE, D. 2015. "Aspectos gramaticales y discursivos de los incisos de cita directa en los textos periodísticos”, en De Lucas Vicente, A., Izquierdo Alegría, D., Jiménez Berrio, F., y Celayeta Gil, N. (eds.), Aplicaciones y enfoques teóricos del Análisis del Discurso. Pamplona: Eunsa, pp. 95-117.

REPEDE, D. 2017. "El uso del discurso reproducido en el corpus PRESEEA-Sevilla: estudio sociolingüístico”. Comunicación presentada en las VI Jornadas Internacionales de Lingüística Hispánica, 3-5 de abril, Lisboa.

REYES, G. 1984. Polifonía textual. La citación en el relato literario. Madrid: Gredos.

REYES, G. 1994. Los procedimientos de cita: citas encubiertas y ecos. Madrid: Arco/Libros.

REYES, G. 2002. Metapragmática. Lenguaje sobre lenguaje, ficciones, figuras. Valladolid: Universidad de Valladolid.

SAN MARTIN, A. 2015. Variación sintáctica y discursiva en el español hablado en Santiago de Chile. Análisis sociolingüístico del queísmo, el discurso referido y los marcadores de reformulación. Tesis doctoral. Valladolid: Universidad de Valladolid. 
SAN MARTIN, A. y GUERRERO, S. 2013. "Una aproximación sociolingüística al empleo del discurso referido en el corpus PRESEEA de Santiago de Chile”, Revista Signos, 46(82), pp. 258-282.

SÁNCHEZ, N. 2010. "Y le dije... Digo...: Análisis del discurso referido e implicaciones para el aula de E/LE”, Monográficos marcoELE, 10, pp. 195-212.

SEARLE, J. 1969. Actos de habla. Ensayo de filosofía del lenguaje. Madrid: Cátedra.

SEMINO, E., SHORT, M., y CULPEPER, J. 1997. "Using a corpus to test and refine a model of speech and thought presentation", Poetics, 25(1), pp. 17-43.

TAGLIAMONTE, S. 2012. Variationist sociolinguistics. Malden: Wiley-Blackwell.

TAGLIAMONTE, S. 2016. "Quantitative analysis in language variation and change", en Sessarego, S. y Tejedo-Herrero, F. (eds.), Spanish language and sociolinguistic analysis. Ámsterdam: John Benjamins, pp. 3-32.

TANNEN, D. 1986. "Introducing constructed dialogue in Greek and American conversational and literary narrative”, en Coulmas, F. (ed.), Direct and indirect speech. Berlin: Mouton de Gruyter, pp. 311-332.

TANNEN, D. 1989. Talking voices: repetition, dialogue and imagery in conversational discourse. Cambridge: Cambridge University Press.

TOGNINI-BONELLI, E. 2004. "Working with corpora: issues and insights", en Coffin, C., Hewings, A., y O’Halloran, K. (eds.), Applying English grammar. Functional and corpus approaches. Londres: Arnold, pp. 11-24.

VAN DER HOUWEN, F. 1998. "Organizing discourse. Direct and indirect speech in Mexican Spanish", Linguistics in the Netherlands, 15, pp. 123-134.

VAN DER HOUWEN, F. 2000. "El habla directa vs. indirecta y la organización del discurso", Foro Hispánico, 17, pp. 27-40.

VARGAS, K. 2014. Estudio de las citas en estilo directo e indirecto en el Corpus sociolingüístico de Caracas 1987. Trabajo de grado de licenciatura. Caracas: Universidad Central de Venezuela.

VERDÍN DÍAZ, G. 1970. Introducción al estilo indirecto libre en español, Revista de Fílología Española, Anejo CXI. Madrid: CSIC.

WILSON, D. 2000. "Metarepresentation in linguistic communication", en Sperber, D. (ed.), Metarepresentations. A multidisciplinary perspective. Oxford: Oxford University Press, pp. 411-448.

\section{*AGRAdECIMIENTOS}

Este estudio ha sido desarrollado en la Eberhard Karls Universität Tübingen, en el marco de una estancia de investigación doctoral financiada por el Servicio Alemán de Intercambio Académico (DAAD). Agradezco a los evaluadores de la revista sus pertinentes comentarios y sugerencias sobre el artículo.

\section{NOTA SOBRE LA AUTORA}

María José Gallucci es profesora agregada en el Departamento de Lenguaje de la Escuela de Letras de la Universidad Central de Venezuela. 